\title{
Glucosyl Hesperidin Lowers Serum Triglyceride Level in Hypertriglyceridemic Subjects through the Improvement of Very Low-Density Lipoprotein Metabolic Abnormality
}

\author{
Yoshikatsu Miwa ${ }^{1}$, Hitoshi Mitsuzumil ${ }^{1, *}$, Takahiro SunAYAmA ${ }^{2}$, Mika YAMADA ${ }^{1}$, \\ Katsuhide OKADA ${ }^{3}$, Michio KUBOTA ${ }^{1}$, Hiroto CHAEN $^{1}$, \\ Yasuo MishIMA ${ }^{4}$ and Masayoshi KiBATA ${ }^{1,5}$ \\ ${ }^{1}$ Hayashibara Biochemical Laboratories, Inc., 1-2-3 Shimoishii, Okayama 700-0907, Japan \\ ${ }^{2}$ Dai-ichi Central Bldg. Clinic, 6-36-601 Honmachi, Okayama 700-0901, Japan \\ ${ }^{3}$ Hayashibara Co., Ltd., 7-7 Amase Minami-machi, Okayama 700-0834, Japan \\ ${ }^{4}$ Tamano Municipal Hospital, 2-3-1 Uno, Tamano, Okayama 706-0011, Japan \\ ${ }^{5}$ National Hospital Organization Minami-Okayama Medical Center \\ 4066 Hayashima, Okayama 701-0304, Japan
}

(Received February 21, 2005)

\begin{abstract}
Summary To examine the serum triglyceride (TG)-lowering effect of a soluble hesperidin derivative, glucosyl hesperidin (G-hesperidin), and its mechanisms, we carried out a G-hesperidin administration test in hypertriglyceridemic subjects. G-Hesperidin was administered to the subjects at $500 \mathrm{mg} / \mathrm{d}$ for $24 \mathrm{wk}$. In this study, the subjects were classified into highTG type (TG $>150 \mathrm{mg} / \mathrm{dL}$ ), borderline-TG type (TG $110-150 \mathrm{mg} / \mathrm{dL}$ ) and normal-TG type (TG $<110 \mathrm{mg} / \mathrm{dL}$ ) on the basis of their initial serum TG values. Among these phenotypes, serum TG level significantly decreased in the high-TG type during the G-hesperidin administration period. It was also observed that elevated values of serum remnant-like particle cholesterol (RLP-C), apolipoprotein (apo) B, apo C-II, apo C-III and apo E occurred in the high-TG type and that these serum levels were significantly reduced by G-hesperidin administration. Moreover, polyacrylamide gel electrophoresis analysis of serum lipoproteins revealed that the very low-density lipoprotein (VLDL)/low-density lipoprotein (LDL) ratio and LDL migration index of the high-TG type were remarkably higher than those of the other phenotypes but that their high values were significantly reduced by the administration. These results indicate that G-hesperidin preferentially lowers serum TG in hypertriglyceridemic subjects and that this effect is possibly caused by the improvement of VLDL metabolic abnormality, leading to the reduction of small dense LDL.
\end{abstract}

Key Words glucosyl hesperidin, hypertriglyceridemia, triglyceride, very low-density lipoprotein, small dense LDL

Hesperidin, which is a flavonoid compound abundantly occurring in citrus fruit peel, is well known to decrease capillary fragility and permeability (1). In addition, this material has been reported to have diverse physiological activities including antioxidant effect (1), antihypertensive effect (2) and antiallergic effect (3). Therefore, hesperidin has attracted attention as a multifunctional food ingredient. However, the use of this flavonoid in the field of foods has been limited because of its low water solubility.

Hijiya and Miyake (4) have synthesized a soluble derivative of hesperidin, glucosyl hesperidin (G-hesperidin), by regioselective transglycosylation with cyclodextrin glucanotransferase from Bacillus stearothermophilus (Fig. 1). G-Hesperidin is very soluble in water, and its solubility is about 10,000 times greater than that of conventional hesperidin (4). Moreover, it has been verified that this derivative releases hesperidin through

${ }^{*}$ To whom correspondence should be addressed.

E-mail: hmitsu@hayashibara.co.jp hydrolysis by brush border $\alpha$-glucosidases $(4,5)$. Our recent studies have shown that G-hesperidin as well as hesperidin exhibits such physiological activities as decrease of serum lipid peroxide level in hyperlipidemic mice (6), antihypertensive effect on spontaneously hypertensive rats $(7,8)$ and suppression of passive cutaneous anaphylaxis in rats (9). These findings indicate that G-hesperidin behaves as hesperidin in the body and results in the exhibition of physiological activities. Thus, G-hesperidin is considered to be available effectively as a hesperidin source.

Furthermore, we have found that the supplementation of G-hesperidin markedly lowers serum triglyceride (TG) in mice with hyperlipidemia induced by a high-fat diet (6). Such a TG-lowering effect has been also observed in humans; it has been confirmed that 6-wk administration of G-hesperidin preferentially lowers serum TG level in hypertriglyceridemic subjects and that this effect is dependent on the dose level and term of G-hesperidin administration (10). It has been demonstrated that an elevated serum TG level is closely associ- 


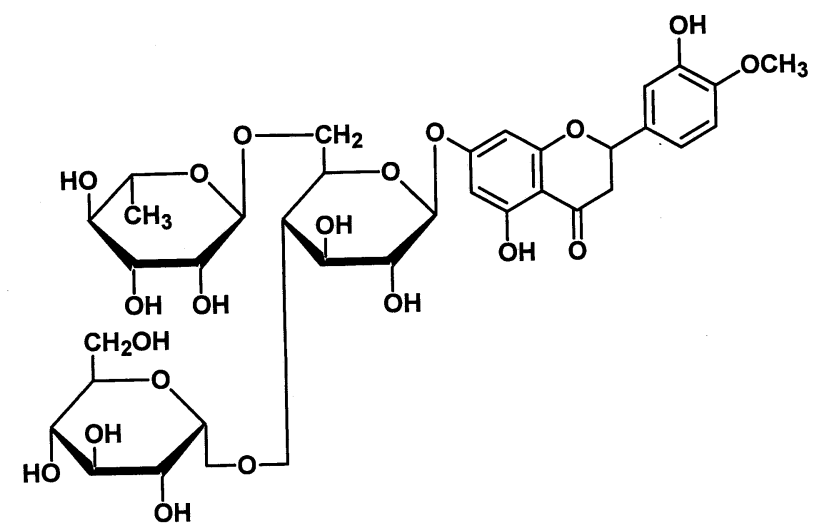

Fig. 1. Chemical structure of glucosyl hesperidin (Ghesperidin).

ated with the development of coronary artery disease and this association is attributed to the formation of small dense low-density lipoprotein (LDL), which has a smaller particle size than normal LDL (11-17). We have also observed that G-hesperidin administration may contribute to the suppression of small dense LDL formation as well as the decrease of serum TG level (10), suggesting a possibility that this flavonoid derivative exhibits a preventive effect against atherosclerosis progression.

In the present study, in order to further understand the effect of G-hesperidin on serum TG and lipoprotein profiles, we carried out a 24-wk administration test of G-hesperidin in hypertriglyceridemic subjects and analyzed the changes in serum lipid parameters.

\section{MATERIALS AND METHODS}

1. Test substances. Tablets consisting of G-hesperidin (250 mg), maltose (750 mg) and sucrose stearate (30 mg) were prepared and used for this study. G-Hesperidin was provided by Toyo Sugar Refining Co., Ltd. (Tokyo, Japan). Maltose was obtained from Hayashibara Biochemical Laboratories, Inc. (Okayama, Japan). Sucrose stearate was purchased from MitsubishiKagaku Foods Corp. (Tokyo, Japan).

2. Subjects. Adult male volunteers, who had a fasting serum TG level of at least $110 \mathrm{mg} / \mathrm{dL}$ and were not receiving any lipid-lowering drugs, were recruited, and 25 men (26-65 y old) were enrolled in this study. The purpose and prospect of the study were fully explained to each volunteer. All subjects gave their informed consent before admission. This study was approved by the Ethics Committee of Hayashibara Biochemical Laboratories, Inc., and conducted in accordance with the Helsinki Declaration of 1975 as revised in 1983.

3. Experimental protocol. After a 4-wk non-treatment baseline period, all subjects received two tablets containing $250 \mathrm{mg}$ of G-hesperidin at each bedtime (500 mg/d G-hesperidin). These tablets were administered for $24 \mathrm{wk}$, followed by a 4 -wk washout period. To examine serum parameters, blood sampling was performed at the beginning of the baseline period (week -4 ), at the beginning of administration (week 0), at

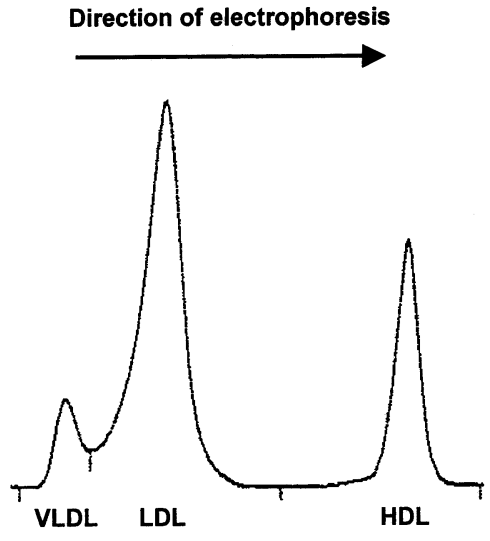

Fig. 2. PAGE densitograph of serum lipoproteins. Serum was subjected to PAGE using the LipoPhor system and then the gel was scanned with a densitometer.

week $4,8,12,16,20$ and 24 of the administration period, and at the end of the washout period (week 28). Blood samples were obtained in the morning after fasting for at least $12 \mathrm{~h}$ from 9 p.m. the previous night. The subjects consumed their usual diet and maintained their eating and exercise habits for the period of this study. To assess their dietary habits, the contents of daily meals and snacks were recorded in the diet diary throughout the study.

4. Serum parameter analyses. Serum TG, total cholesterol (TC), LDL-cholesterol (LDL-C) and high-density lipoprotein-cholesterol (HDL-C) were measured using an automated analyzer. Remnant-like particle cholesterol (RLP-C) was measured by the immunoadsorption method of Nakajima et al. (18). Serum apolipoprotein (apo) A-I, B, C-II, C-III and E were measured by immunoturbidimetry. Serum aspartate aminotransaminase (AST), alanine aminotransaminase (ALT) and $\gamma$ glutamyl-transferase $(\gamma$-GT) were determined in accordance with the methods standardized by Japan Society of Clinical Chemistry $(19,20)$. Moreover, serum creatinine and uric acid were measured by the Jaffe method (21) and an uricase-peroxidase method (22), respectively. All the analyses were performed by FALCO Biosystems Ltd. (Okayama, Japan).

5. Analysis of serum lipoprotein composition and evaluation of LDL particle size. Serum lipoproteins were fractionated by polyacrylamide gel electrophoresis (PAGE) using the LipoPhor system (Jokoh Co., Ltd., Tokyo, Japan) as described by Mishima et al. (23-26). Briefly, fresh serum was electrophoresed on polyacrylamide gel containing Sudan Black B and then the gel was scanned with a densitometer. Figure 2 shows typical pattern of PAGE densitograph. From the PAGE pattern thus obtained, the ratio of the peak height of very lowdensity lipoprotein (VLDL) fraction to one of LDL fraction was calculated (VLDL/LDL ratio). The change in serum lipoprotein composition was analyzed based on the VLDL/LDL ratio. Moreover, the distance between the peaks of VLDL and LDL fractions and the distance between the peaks of VLDL and HDL fractions were measured on the PAGE pattern, and the relative migra- 
tion index of LDL fraction (LDL-MI) was calculated by the following formula:

$\mathrm{LDL}-\mathrm{MI}=($ the distance between the peaks of VLDL and LDL fractions)/(the distance between the peaks of VLDL and HDL fractions)

This value is correlated with LDL particle size (23-26). That is to say, the increase of LDL-MI indicates the decrease of LDL particle size. In particular, when the value is more than 0.35 , the presence of small dense LDL is speculated (26).

6. Classification of subjects according to hypertriglyceridemia phenotypes and efficacy analyses in each phenotype. In each serum parameter analyses, the mean of the two measurements obtained during the baseline period (the mean of week -4 and week 0 values) was regarded as the initial value. Based on the initial serum TG value thus obtained, all subjects were classified into the following 3 phenotypes: high-TG type (TG >150 $\mathrm{mg} / \mathrm{dL}$ ), borderline-TG type (TG 110-150 mg/dL) and normal-TG type (TG $<110 \mathrm{mg} / \mathrm{dL}$ ). The criteria for the phenotype classification were determined according to the descriptions of Ai and Tanaka (27) and Vigna et al. (28). To assess the efficacies of G-hesperidin, the changes of the initial values in serum parameters were analyzed in each phenotype at week $4,8,12,16,20$, 24 and 28. Additionally, the changes of the initial values in VLDL/LDL ratio and LDL-MI were analyzed in the individual phenotypes at week 12, 24 and 28.

7. Statistical analyses. Data were expressed as means \pm SD. The initial value (the mean of week -4 and week 0 values) was considered the baseline, and the change from the baseline in each group was examined by the Wilcoxon signed-ranks test. Differences in the initial values of respective parameters among groups were examined by one-way ANOVA. The significance level was set at $p<0.05$ for all tests. Statistical analyses were performed with Stat View version 5.0 for Windows (SAS Institute, Cary, NC).

\section{RESULTS}

1. Classification of subjects and their characteristics in each hypertriglyceridemia phenotype

To investigate the efficacies of G-hesperidin administration, we classified the study subjects into the 3 phenotypes (high-TG type, borderline-TG type and normalTG type) on the basis of their initial serum TG values. Table 1 shows baseline characteristics of the subjects in each phenotype. The subjects were divided into 8 of high-TG type, 11 of borderline-TG type and 6 of normal-TG type, respectively, of which the initial serum TG values were in turns $226.5 \pm 89.9,128.8 \pm 13.8$ and $82.5 \pm 19.8 \mathrm{mg} / \mathrm{dL}$. When the initial values of the other parameters were compared among the 3 phenotypes, serum RLP-C, apo B, apo C-II, apo C-III, apo E, AST, ALT and $\gamma$-GT values tended to become high with the elevation of serum TG level. A similar tendency was also observed for the VLDL/LDL ratio and LDL-MI.

No change was recognized in dietary habits with any

Table 1. Baseline characteristics of the subjects in each hypertriglyceridemia phenotype.

\begin{tabular}{|c|c|c|c|}
\hline & High-TG type & Borderline-TG type & Normal-TG type \\
\hline Number of subjects & 8 & 11 & 6 \\
\hline Age (y) & $43.8 \pm 11.4^{\mathrm{a}}$ & $46.9 \pm 9.6^{\mathrm{a}}$ & $39.3 \pm 11.6^{\mathrm{a}}$ \\
\hline $\mathrm{TG}(\mathrm{mg} / \mathrm{dL})$ & $226.5 \pm 89.9^{a}$ & $128.8 \pm 13.8^{b}$ & $82.5 \pm 19.8^{b}$ \\
\hline RLP-C (mg/dL) & $11.0 \pm 7.2^{\mathrm{a}}$ & $5.1 \pm 0.8^{b}$ & $3.7 \pm 0.5^{b}$ \\
\hline $\mathrm{TC}(\mathrm{mg} / \mathrm{dL})$ & $241.3 \pm 39.3^{\mathrm{a}}$ & $240.9 \pm 32.8^{a}$ & $226.7 \pm 26.6^{\mathrm{a}}$ \\
\hline $\mathrm{LDL}-\mathrm{C}(\mathrm{mg} / \mathrm{dL})$ & $148.4 \pm 33.7^{\mathrm{a}}$ & $158.0 \pm 30.3^{a}$ & $155.4 \pm 21.9^{\mathrm{a}}$ \\
\hline HDL-C (mg/dL) & $49.6 \pm 10.3^{a}$ & $52.5 \pm 13.2^{\mathrm{a}}$ & $53.2 \pm 7.2^{\mathrm{a}}$ \\
\hline Apo A-I (mg/dL) & $157.6 \pm 28.5^{\mathrm{a}}$ & $151.3 \pm 29.7^{\mathrm{a}}$ & $145.7 \pm 12.7^{\mathrm{a}}$ \\
\hline Apo B (mg/dL) & $142.3 \pm 23.8^{\mathrm{a}}$ & $138.4 \pm 22.6^{\mathrm{a}}$ & $124.9 \pm 15.9^{\mathrm{a}}$ \\
\hline Apo C-II (mg/dL) & $8.3 \pm 2.0^{\mathrm{a}}$ & $6.6 \pm 1.8^{\mathrm{a}}$ & $4.5 \pm 0.8^{b}$ \\
\hline Apo C-III $(\mathrm{mg} / \mathrm{dL})$ & $18.6 \pm 4.3^{\mathrm{a}}$ & $14.3 \pm 4.7^{\mathrm{a}, \mathrm{b}}$ & $9.4 \pm 1.1^{\mathrm{b}}$ \\
\hline Apo E (mg/dL) & $6.6 \pm 1.1^{\mathrm{a}}$ & $5.7 \pm 1.2^{\mathrm{a}, \mathrm{b}}$ & $4.9 \pm 0.5^{b}$ \\
\hline VLDL/LDL ratio & $0.66 \pm 0.20^{\mathrm{a}}$ & $0.47 \pm 0.10^{\mathrm{b}}$ & $0.33 \pm 0.08^{\mathrm{b}}$ \\
\hline LDL-MI & $0.362 \pm 0.019^{\mathrm{a}}$ & $0.351 \pm 0.012^{\mathrm{a}}$ & $0.327 \pm 0.010^{b}$ \\
\hline AST (IU/L) & $31.3 \pm 9.5^{a}$ & $30.0 \pm 13.7^{\mathrm{a}}$ & $23.3 \pm 9.1^{\mathrm{a}}$ \\
\hline ALT (IU/L) & $49.1 \pm 31.7^{\mathrm{a}}$ & $37.5 \pm 28.5^{\mathrm{a}}$ & $33.2 \pm 35.2^{\mathrm{a}}$ \\
\hline$\gamma-\mathrm{GT}(\mathrm{IU} / \mathrm{L})$ & $114.7 \pm 78.6^{\mathrm{a}}$ & $71.7 \pm 68.9^{\mathrm{a}}$ & $39.1 \pm 30.3^{\mathrm{a}}$ \\
\hline Creatinine $(\mathrm{mg} / \mathrm{dL})$ & $1.0 \pm 0.1^{\mathrm{a}}$ & $1.0 \pm 0.1^{\mathrm{a}}$ & $1.0 \pm 0.1^{\mathrm{a}}$ \\
\hline Uric acid (mg/dL) & $6.2 \pm 0.7^{a}$ & $6.0 \pm 0.9^{a}$ & $5.8 \pm 0.8^{\mathrm{a}}$ \\
\hline Body weight (kg) & $72.2 \pm 7.7^{\mathrm{a}}$ & $68.9 \pm 9.2^{\mathrm{a}}$ & $67.8 \pm 14.3^{\mathrm{a}}$ \\
\hline Body mass index & $25.1 \pm 2.3^{\mathrm{a}}$ & $24.1 \pm 2.6^{\mathrm{a}}$ & $24.0 \pm 3.6^{\mathrm{a}}$ \\
\hline
\end{tabular}

In each parameter, the mean of the two measurements obtained at week -4 and week 0 was regarded as the initial value. Data in the table represent the initial values of respective parameters (means \pm SD). The subjects were classified into the 3 phenotypes on the basis of their initial serum TG values: high-TG type (TG $>150 \mathrm{mg} / \mathrm{dL}$ ), borderline-TG type (TG 110$150 \mathrm{mg} / \mathrm{dL}$ ) and normal-TG type (TG $<110 \mathrm{mg} / \mathrm{dL}$ ). Data not sharing a common letter in the same row are significantly different at $p<0.05$. 


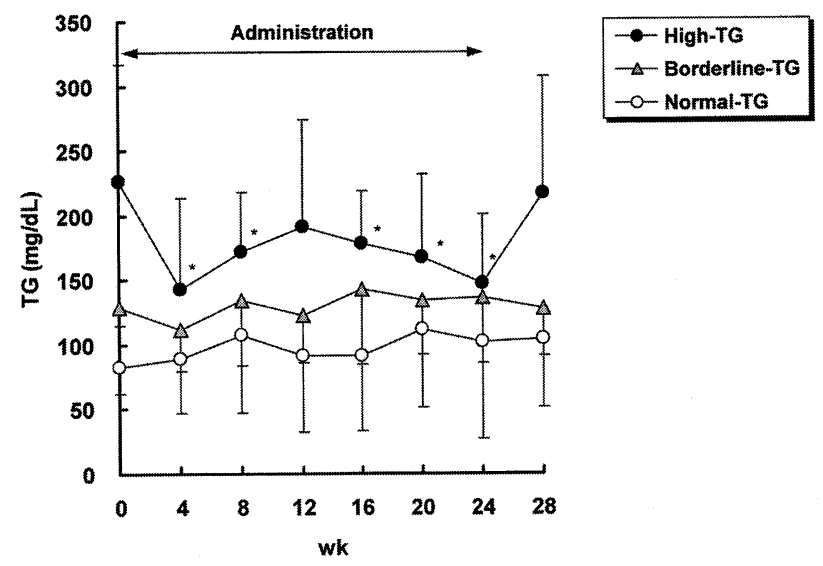

Fig. 3. Changes in serum TG level of respective phenotypes. Tablets containing $250 \mathrm{mg}$ of G-hesperidin were administered at the prescribed dose (two tablets once daily) for $24 \mathrm{wk}$. Blood sampling was performed at week $-4,0,4,8,12,16,20,24$ and 28 , and serum TG level was measured at each time point. The mean of the two measurements obtained at week -4 and week 0 was regarded as the initial serum TG value. This value is represented as the week 0 value in the figure. Data are means $\pm \mathrm{SD}$ of respective phenotype subjects. ${ }^{*} p<0.05$ vs the initial value.

of the subjects throughout the study. There was no change in body weight or body mass index in any phenotype during the study. Furthermore, there were no changes in serum creatinine or uric acid levels in any phenotype during the administration period. No clinical signs related to the treatment were observed in any phenotype.

2. Change in serum TG level

In the individual phenotypes mentioned above, we analyzed the changes in serum TG level after G-hesperidin administration. The result is shown in Fig. 3.

No obvious changes in serum TG level were observed in the borderline-TG or normal-TG types during the study.

On the other hand, the high-TG type subjects had a significant reduction in serum TG level after week 4 , and the effect was maintained throughout the administration period. At week 24, serum TG value decreased to $147.0 \pm 53.8 \mathrm{mg} / \mathrm{dL}(-34 \%)$, which was nearly within the normal range. Additionally, this decrease was abrogated by the cessation of administration.

3. Change in serum RLP-C level

Serum TG level is known to be correlated with the serum concentrations of VLDL and remnant lipoproteins generated in the catabolic process of VLDL (29). Therefore, we measured serum RLP-C as an index of serum remnant lipoprotein concentrations and analyzed whether it is altered by G-hesperidin administration in each phenotype. The result is shown in Fig. 4.

The initial serum RLP-C value of the high-TG type was higher than those of the borderline-TG and normal-TG types.

Although the borderline-TG and normal-TG type subjects showed some changes in serum RLP-C level
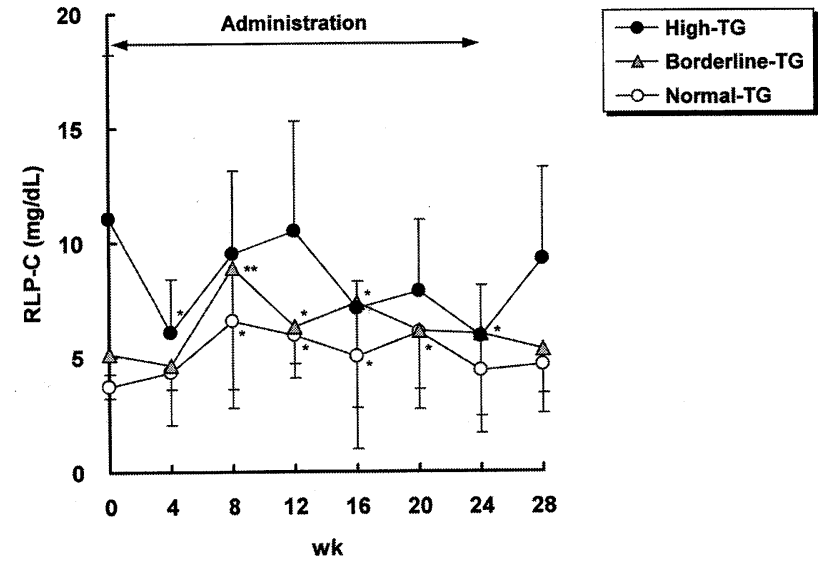

Fig. 4. Changes in serum RLP-C level of respective phenotypes. Tablets containing $250 \mathrm{mg}$ of G-hesperidin were administered at the prescribed dose (two tablets once daily) for $24 \mathrm{wk}$. Blood sampling was performed at week $-4,0,4,8,12,16,20,24$ and 28 , and serum RLP-C level was measured at each time point. The mean of the two measurements obtained at week -4 and week 0 was regarded as the initial serum RLP-C value. This value is represented as the week 0 value in the figure. Data are means \pm SD of respective phenotype subjects. ${ }^{*} p<0.05$ vs the initial value, ${ }^{* *} p<0.01$ vs the initial value.

during the study, such variations were limited within the normal range $(\leqq 7.5 \mathrm{mg} / \mathrm{dL})$. Thus, no obvious changes in serum RLP-C level after G-hesperidin administration were detected in these phenotype subjects.

On the other hand, the high-TG type subjects, who showed no obvious change in serum RLP-C level during the first half of the administration period, had a steady reduction in it during the latter half of the treatment. After week 16, serum RLP-C level decreased to the normal range, and the effect had a tendency to disappear during the washout period.

From these results, the profiles of serum RLP-C changes were found to agree well with those of serum TG changes in individual phenotypes.

4. Changes in serum TC, LDL-C and HDL-C levels

The changes in serum TC, LDL-C and HDL-C levels after G-hesperidin administration examined in each phenotype are shown in Fig. 5.

There were no differences in any of the initial serum TC, LDL-C and HDL-C values among the 3 phenotypes.

Serum TC and LDL-C levels tended to be gradually reduced in every phenotype during the administration period. In particular, they were kept low after week 16 and decreased to nearly the normal ranges at week 24 . Furthermore, these effects had a tendency to disappear with the cessation of administration.

In contrast, serum HDL-C level showed no obvious change in any phenotype during the administration period.

\section{Changes in serum apolipoprotein levels}

In each phenotype, we also investigated whether the serum levels of 5 apolipoproteins (apo A-I, B, C-II, C-III and $\mathrm{E}$ ), which are correlated with serum lipids, were 
TC

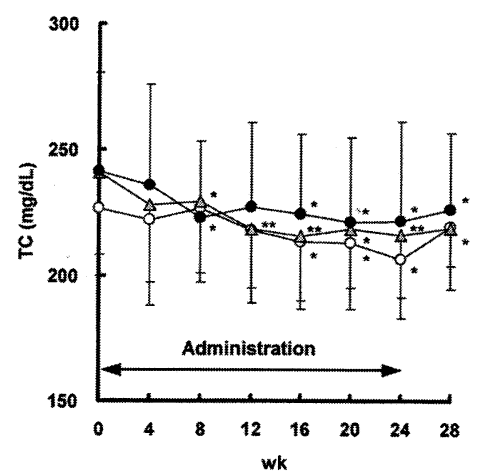

LDL-C

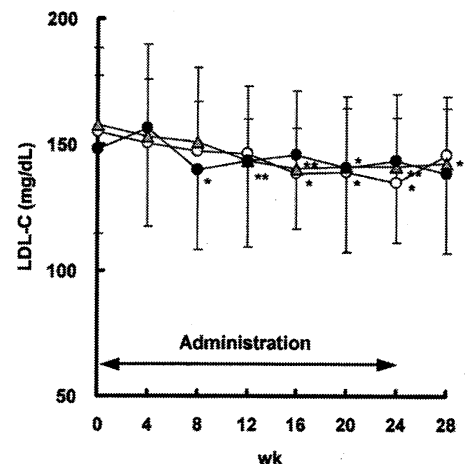

HDL-C

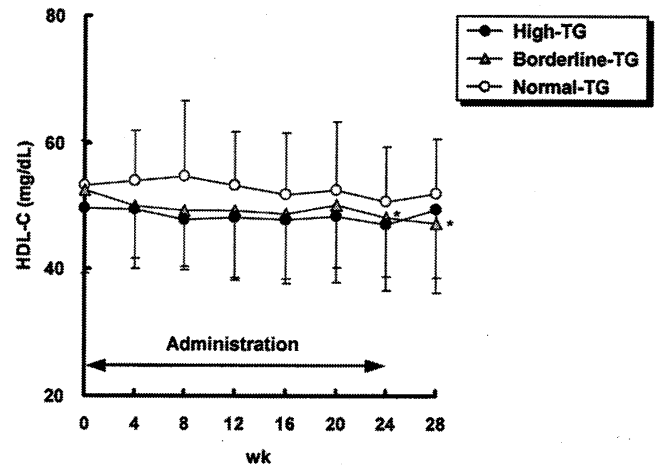

Fig. 5. Changes in serum TC, LDL-C and HDL-C levels of respective phenotypes. Tablets containing 250 mg of G-hesperidin were administered at the prescribed dose (two tablets once daily) for $24 \mathrm{wk}$. Blood sampling was performed at week $-4,0$, $4,8,12,16,20,24$ and 28 , and serum cholesterol levels were measured at each time point. The mean of the two measurements obtained at week -4 and week 0 was regarded as the initial value in each serum cholesterol analysis. This value is represented as the week 0 value in each graph. Data are means \pm SD of respective phenotype subjects. ${ }^{*} p<0.05$ vs the initial value, ${ }^{* *} p<0.01$ vs the initial value.

Apo A-I

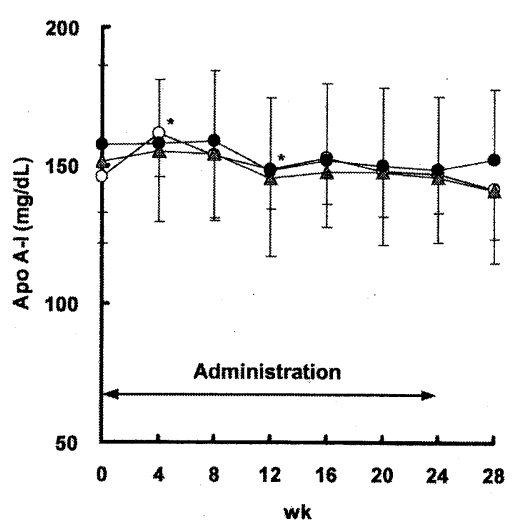

Apo C-III

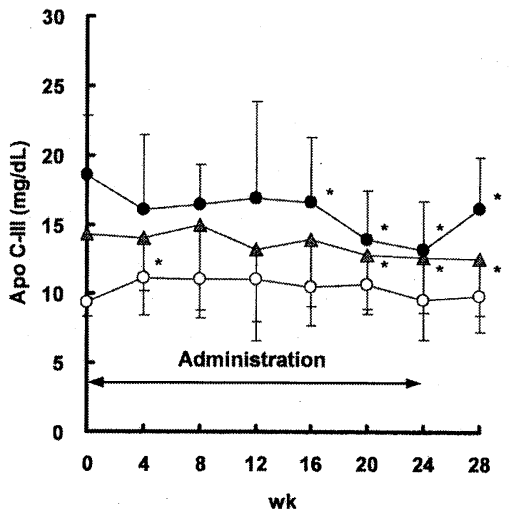

Apo B

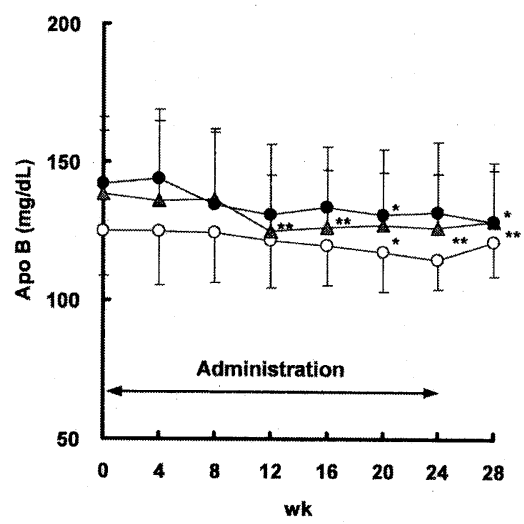

Apo E

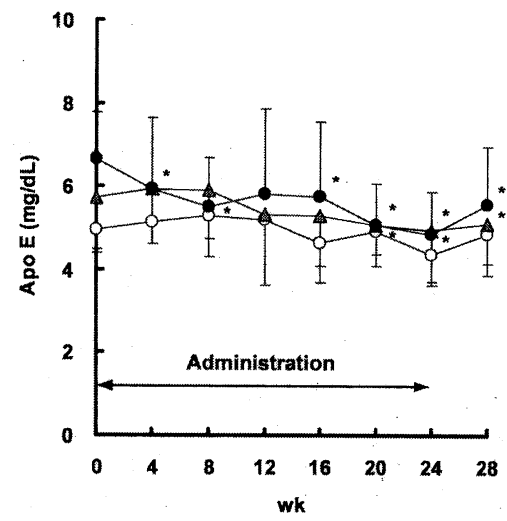

Apo C-II

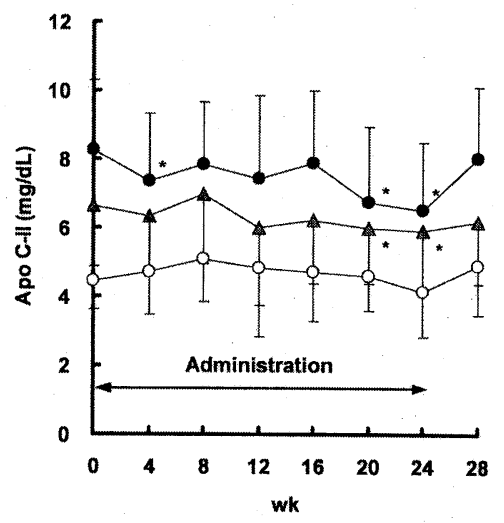

Fig. 6. Changes in serum apolipoprotein levels of respective phenotypes. Tablets containing $250 \mathrm{mg}$ of G-hesperidin were administered at the prescribed dose (two tablets once daily) for $24 \mathrm{wk}$. Blood sampling was performed at week -4, 0, 4, 8, $12,16,20,24$ and 28, and serum apolipoprotein levels were measured at each time point. The mean of the two measurements obtained at week -4 and week 0 was regarded as the initial value in each serum apolipoprotein analysis. This value is represented as the week 0 value in each graph. Data are means \pm SD of respective phenotype subjects. ${ }^{*} p<0.05$ vs the initial value, ${ }^{* *} p<0.01$ vs the initial value. 

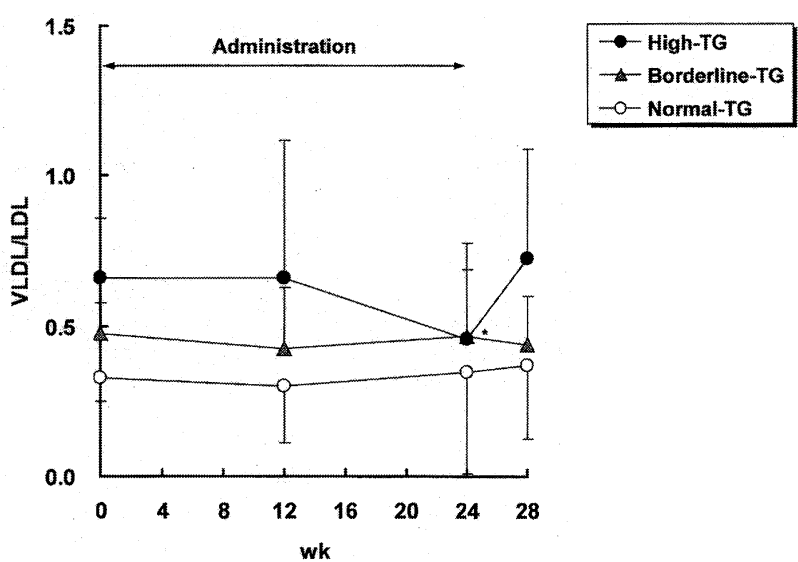

Fig. 7. Changes in VLDL/LDL ratio of respective phenotypes. Tablets containing $250 \mathrm{mg}$ of G-hesperidin were administered at the prescribed dose (two tablets once daily) for $24 \mathrm{wk}$. Blood sampling was performed at week $-4,0,12,24$ and 28. Serum was subjected to PAGE using the LipoPhor system at each time point, and VLDL/LDL ratio was calculated from the densitograph. The mean of the two measurements obtained at week -4 and week 0 was regarded as the initial VLDL/ LDL ratio. This value is represented as the week 0 value in the figure. Data are means \pm SD of respective phenotype subjects. ${ }^{*} p<0.05$ vs the initial value. altered by G-hesperidin administration. The result is shown in Fig. 6.

No differences were seen in the initial serum apo A-I value among the 3 phenotypes. Moreover, this serum level showed no obvious change in any phenotype during the administration period.

The initial serum apo B, C-II, C-III and E values tended to be elevated in the high-TG type, compared to those of the borderline-TG and normal-TG types. Elevated values of these apolipoproteins significantly decreased in the high-TG type during the administration period; particularly, they were markedly reduced in the latter half of the period. These effects had a tendency to disappear $4 \mathrm{wk}$ after the cessation of administration. In addition, similar effects were also observed in the borderline-TG type subjects, but they failed to be observed in the normal-TG type.

6. Change in serum lipoprotein composition

In the individual phenotypes, the changes in serum lipoprotein composition after G-hesperidin administration were analyzed on the basis of the VLDL/LDL ratio, which was calculated on the PAGE pattern of serum lipoproteins. The result is shown in Fig. 7. Additionally, typical PAGE patterns obtained in this analysis are shown in Fig. 8.

The initial VLDL/LDL ratio of the high-TG type was higher than those of the borderline-TG and normal-TG
Week 0
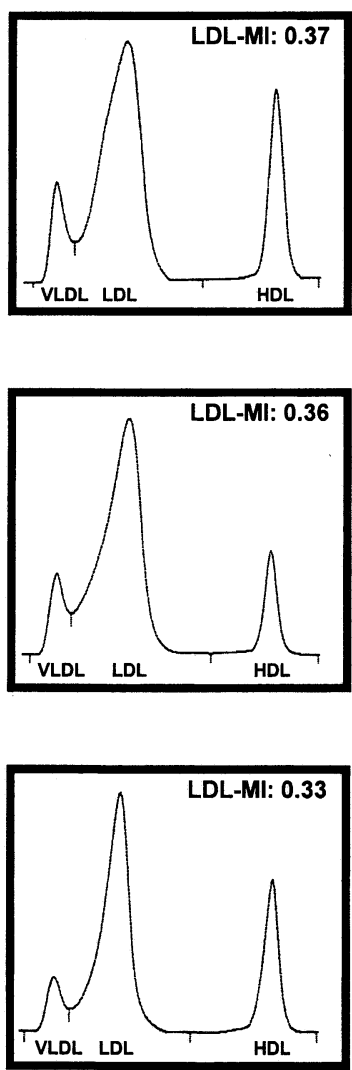

Week 24
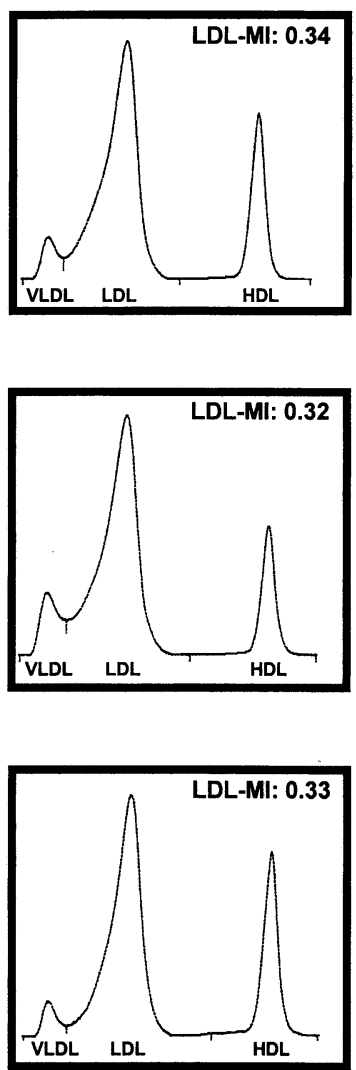

Week 28
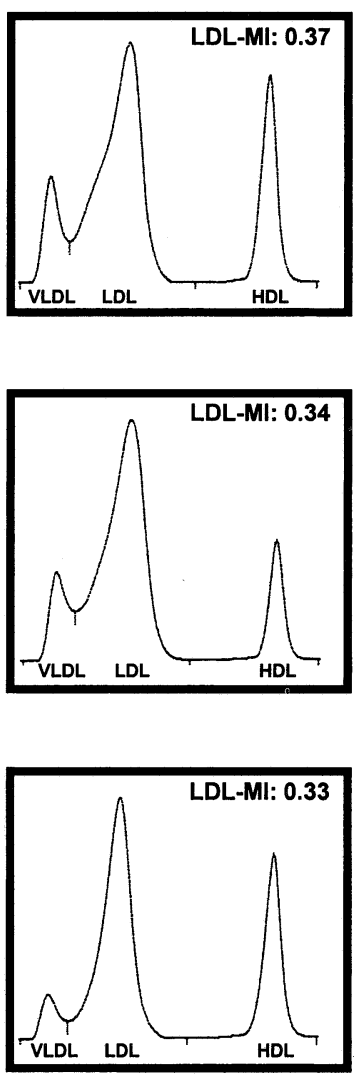

Fig. 8. Typical PAGE patterns of serum lipoproteins in respective phenotypes and their changes by G-hesperidin administration. The experiment was performed as described in Fig. 7, and serum obtained at the indicated time point was subjected to PAGE using the LipoPhor system. LDL-MI was calculated on each PAGE pattern. 

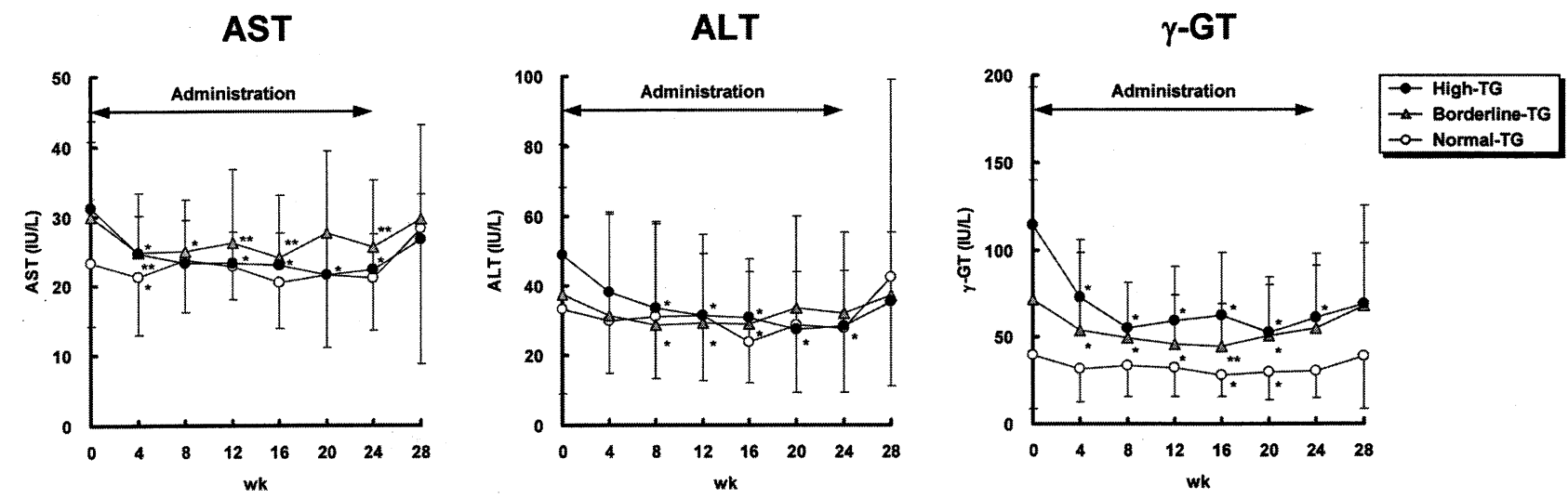

Fig. 9. Changes in serum AST, ALT and $\gamma$-GT levels of respective phenotypes. Tablets containing $250 \mathrm{mg}$ of G-hesperidin were administrated at the prescribed dose (two tablets once daily) for $24 \mathrm{wk}$. Blood sampling was performed at week -4 , $0,4,8,12,16,20,24$ and 28, and serum AST, ALT and $\gamma$-GT levels were measured at each time point. The mean of the two measurements obtained at week -4 and week 0 was regarded as the initial value in each serum parameter analysis. This value is represented as the week 0 value in each graph. Data are means \pm SD of respective phenotype subjects. ${ }^{*} p<0.05$ vs the initial value, ${ }^{* *} p<0.01$ vs the initial value.

types. From this observation, the rate of VLDL accumulation in serum was suggested to be high in the high-TG type as compared with the other phenotypes.

The borderline-TG and normal-TG types showed no obvious alteration in the VLDL/LDL ratio during the study.

In contrast, the high-TG type had a significant reduction in the VLDL/LDL ratio during the administration period, suggesting that the rate of serum VLDL accumulation was lowered to the same level as in the borderline-TG and normal-TG types. This effect was abrogated during the washout period.

7. Changes in serum AST, ALT and $\gamma$-GT levels

The influences of G-hesperidin administration on hepatic functions in each phenotype were further examined on the basis of serum AST, ALT and $\gamma$-GT values. The result is shown in Fig. 9.

The initial serum AST, ALT and $\gamma$-GT values of the high-TG type tended to be higher than those of the borderline-TG and normal-TG types.

The normal-TG type had no obvious changes in serum AST, ALT or $\gamma$-GT levels during the study.

On the other hand, the high-TG type had a significant reduction in these serum levels during the administration period. In particular, serum ALT and $\gamma$-GT levels were remarkably reduced after G-hesperidin administration in this phenotype. These effects were abrogated during the washout period. In addition, similar effects were also observed in the borderline-TG type, although they were mild as compared with the effects obtained in the high-TG type.

These observations suggest that in the subjects with a high serum TG level their hepatic functions are improved by G-hesperidin administration.

8. Change in LDL particle size

In respective phenotypes, we further analyzed the changes in LDL particle size on the basis of the LDL-MI, which was calculated on the PAGE pattern of serum lipoproteins. The result is shown in Fig. 10 (see Fig. 8 about typical PAGE patterns).

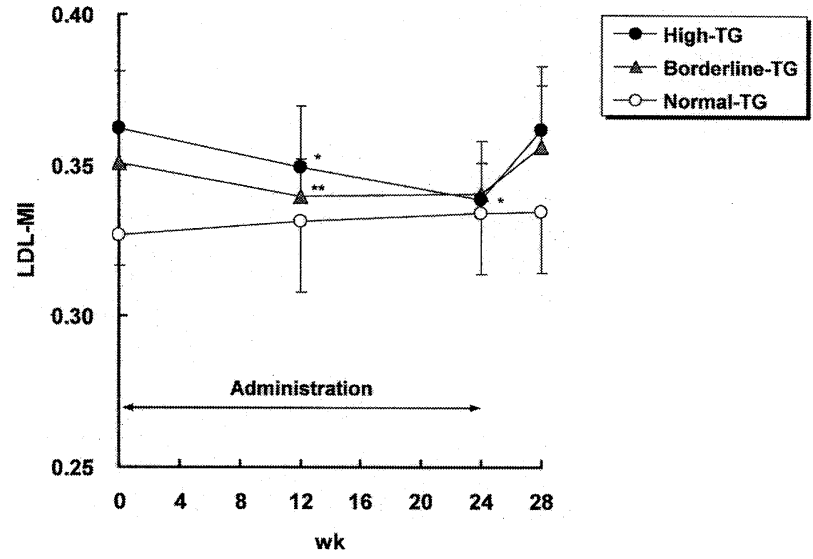

Fig. 10. Changes in LDL-MI of respective phenotypes. Tablets containing $250 \mathrm{mg}$ of G-hesperidin were administered at the prescribed dose (two tablets once daily) for $24 \mathrm{wk}$. Blood sampling was performed at week $-4,0,12,24$ and 28. Serum was subjected to PAGE using the LipoPhor system at each time point, and LDLMI was calculated from the densitograph. The mean of the two measurements obtained at week -4 and week 0 was regarded as the initial LDL-MI. This value is represented as the week 0 value in the figure. Data are means \pm SD of respective phenotype subjects. ${ }^{*} p<0.05$ vs the initial value, ${ }^{* *} p<0.01$ vs the initial value.

The initial LDL-MI of the high-TG type tended to be higher than those of the borderline-TG and normal-TG types, and the value was more than 0.35. From this observation, the LDL particle size of the high TG type was suggested to be smaller than those of the other phenotypes. Additionally, it was speculated that the highTG type might possess small dense LDL.

The LDL-MI of the normal-TG type was unchanged throughout the study and remained within the normal range $(<0.35)$.

In contrast, the LDL-MI of the high-TG type was significantly reduced during the administration period, indicating an expansion of LDL particle size. This effect 
disappeared $4 \mathrm{wk}$ after the cessation of administration. Furthermore, a similar effect was also recognized in the borderline-TG type, although it was moderate as compared with the effect obtained in the high-TG type.

These results indicate that the subjects with a high serum TG level, especially the high-TG type subjects, are led to the reduction of small dense LDL formation by Ghesperidin administration.

\section{DISCUSSION}

Our previous study has shown that G-hesperidin preferentially lowers serum TG level in hypertriglyceridemic subjects and may contribute to the suppression of small dense LDL formation (10). In the present study, to gain a better understanding of these effects by G-hesperidin and their mechanisms, we examined whether 24-wk administration of G-hesperidin affects serum lipid parameters in subjects whose serum TG level is more than $110 \mathrm{mg} / \mathrm{dL}$.

As shown in Table 1, the study subjects were classified into high-TG type (TG $>150 \mathrm{mg} / \mathrm{dL}$ ), borderline-TG type (TG $110-150 \mathrm{mg} / \mathrm{dL}$ ) and normal-TG type (TG $<110 \mathrm{mg} / \mathrm{dL})$. Among these phenotypes, serum TG level showed a significant reduction in the high-TG type during the administration period (Fig. 3). This result agreed well with that obtained by the 6 -wk administration test of G-hesperidin, which was conducted in our previous study (10). From these observations, therefore, it was reconfirmed that G-hesperidin is effective in cases of elevated serum TG level.

Hypertriglyceridemia is known to be attributed to the accumulation of serum TG-rich lipoproteins such as VLDL and its metabolites, remnant lipoproteins (3032). In addition, it is considered that the accumulation of these TG-rich lipoproteins in serum is caused by an unbalance of the synthesis and catabolism of VLDL, namely, VLDL metabolic abnormality (30-32). In the present test, it was observed that the initial serum RLP$\mathrm{C}$ value of the high-TG type was markedly elevated in comparison with those of the other phenotypes (Fig. 4). This implies that the accumulation of serum remnant lipoproteins occurs in the high-TG type subjects. Moreover, the high-TG type subjects had a significant reduction in serum RLP-C level during the G-hesperidin administration period (Fig. 4), suggesting that G-hesperidin decreases the accumulation of serum remnant lipoproteins. Therefore, there is a possibility that G-hesperidin improves VLDL metabolic abnormality in the high-TG type. This effect is likely to be elicited by the suppression of VLDL synthesis or the augmentation of VLDL catabolism.

The possibility mentioned above was supported by the results of serum apolipoprotein analyses. We observed that elevated values of serum apo C-II, apo CIII and apo E occurred in the high-TG type and that these apolipoprotein levels were significantly reduced by G-hesperidin administration (Fig. 6). Apo C-II, apo C-III and apo $\mathrm{E}$ are bound to VLDL and remnant lipoproteins, respectively (33-35); and furthermore, their high values reflect the accumulation of serum VLDL and rem- nant lipoproteins. Our results indicate that the accumulation of serum VLDL and remnant lipoproteins occurs in the high-TG type and that these serum levels are reduced by G-hesperidin administration.

The effect of G-hesperidin on VLDL metabolic abnormality was further clarified by the PAGE analysis of serum lipoproteins. When serum lipoprotein composition of respective phenotypes was investigated using PAGE analysis, the initial VLDL/LDL ratio of the highTG type was remarkably higher than those of the other phenotypes (Figs. 7 and 8). This result indicates that the rate of VLDL accumulation in serum is elevated in the high-TG type as compared with the other phenotypes, probably reflecting an excess of VLDL synthesis or a deficiency of VLDL catabolism. In the high-TG type subjects with such VLDL metabolic abnormality, G-hesperidin significantly lowered their VLDL/LDL ratios (Figs. 7 and 8). This observation provides proof that Ghesperidin improves the metabolic abnormality in the high-TG type through the suppression of VLDL synthesis or the augmentation of VLDL catabolism.

It is not clear whether G-hesperidin acts on the synthetic process or the catabolic process of VLDL. However, we observed that serum apo B level was significantly reduced during the administration period (Fig. 6). It is known that apo B forms VLDL by complexing with TG and cholesteryl ester in hepatocytes and is secreted from the liver (36-39). On the basis of this finding, serum apo B level is regarded as an index of hepatic VLDL synthesis. Thus, our observation may be possibly interpreted to mean that G-hesperidin suppresses VLDL synthesis in the liver. In addition, we recently found that G-hesperidin significantly decreased apo B secretion in cultured human hepatocytes (unpublished data). Moreover, hesperetin, which is released from hesperidin through deglycosylation by bacteria in the gastrointestinal tract, has been reported to inhibit apo B secretion in cultured human hepatocytes (40, 41). These findings strongly suggest a possibility that Ghesperidin suppresses hepatic VLDL synthesis. Hence, in vitro experiments monitoring apo B secretion from hepatocytes are in progress in our laboratory to elucidate the influence of G-hesperidin on hepatic VLDL synthesis.

Interestingly, it was observed that the initial serum AST, ALT and $\gamma$-GT values of the high-TG type tended to be higher than those of the other phenotypes and that these serum levels were drastically lowered during the administration period (Fig. 9). From these results, G-hesperidin is suggested to repair the abnormal hepatic functions of the high-TG type subjects. Additionally, these observations support that G-hesperidin exhibits its effects in the liver. As mentioned above, VLDL is synthesized via the assembly of TG, cholesteryl ester and apo B in the liver and secreted into blood circulation (36-39). Therefore, it seems likely that G-hesperidin acts on the process of VLDL synthesis in the liver. In order to further clarify this, we recently started the investigations focused on the process of VLDL assembly in the liver. On the other hand, it has been 
reported that hesperetin reduces the hepatic TG accumulation in orotic acid-fed rats through the inhibition of a TG synthetic enzyme, phosphatidic acid phosphohydrolase (42). Thus, G-hesperidin may also exhibit similar effect in the liver.

Although we previously observed that serum TC and LDL-C levels were not altered by 6 -wk administration of G-hesperidin (10), these serum levels significantly decreased after week 16 of the administration period in the present study (Fig. 5). Since the decrease of these serum cholesterol levels was detected in all phenotypes (Fig. 5), this effect was speculated to be caused independently of the mechanisms by which G-hesperidin lowered serum TG level. It has been reported that hesperidin lowers serum TC and LDL-C levels in rats and that the effect is attributed to suppressing cholesterol synthesis by the inhibition of 3-hydroxy-3-methylglutaryl coenzyme A (HMG-CoA) reductase $(43,44)$. Such a mechanism was considered to cause G-hesperidin to lower serum TC and LDL-C levels of the subjects in this study. However, it seems likely that long-term administration is required to lower these serum cholesterol levels. Human HMG-CoA reductase may have a low susceptibility to G-hesperidin, compared to that of rats.

In contrast, the serum HDL-C level showed no obvious change from G-hesperidin administration (Fig. 5) as described previously (10). Moreover, the serum level of apo A-I, bound to HDL, was also unchanged during the administration period (Fig. 6). HDL is recognized to play a critical role in the process of VLDL catabolism because it removes TG from VLDL via cholesteryl ester transfer protein (CETP) $(45,46)$. Nevertheless, on the basis of our observations, it is unlikely that G-hesperidin enhances VLDL catabolism through affecting the TG removal by CETP.

Recent studies have shown that hypertriglyceridemic patients possess small dense LDL with high frequency $(14,15)$ and that this abnormal LDL is closely involved in coronary artery disease $(16,17)$. In addition, it has been presumed that small dense LDL formation, which remains unclear, can be attributed to the accumulation of serum TG-rich lipoproteins by VLDL metabolic abnormality (47-49). As expected, LDL particle size and serum level of TG or RLP-C tended to be inversely correlated in the preset study (Table 1, Fig. 10). Especially, the initial LDL particle size of the high-TG type was smaller than those of the other phenotypes, suggesting the presence of small dense LDL (Figs. 8 and 10). In this phenotype, G-hesperidin administration led to an increase in LDL particle size (Figs. 8 and 10). This result is in good agreement with that reported previously (10). From these findings, it is suggested that G-hesperidin improves VLDL metabolic abnormality and results in the reduction of small dense LDL formation.

The active form of G-hesperidin in vivo is considered to be hesperetin. In fact, Chiba et al. have reported that hesperetin conjugated with glucuronic acid is detected in the serum of mice fed G-hesperidin (50). Furthermore, we have observed that serum concentrations of hesperetin glucuronides in rats administered G-hesperi- din are markedly higher than those in rats administered hesperidin (6). This observation implies that G-hesperidin is absorbed more efficiently than hesperidin due to its high water solubility. Based on such a beneficial property, G-hesperidin can be assumed to exhibit its activity effectively in the body.

In conclusion, the present study has indicated that a 24-wk administration of G-hesperidin preferentially lowers serum TG level in hypertriglyceridemic subjects and that this effect is possibly due to the repair of VLDL metabolic abnormality. This action also participates in the suppression of small dense LDL formation, suggesting a potential for preventing atherosclerosis progression.

\section{Acknowledgments}

We would like to thank Ms. Michiyo Tsujita for her excellent technical and secretarial assistance in this study.

This work was supported in part by the Technical Development Program for Agribusiness through Utilizing Concentrated Know-how from the Private Sector, from the Ministry of Agriculture, Forestry and Fisheries of Japan.

\section{REFERENCES}

1) Garg A, Garg S, Zaneveld LJD, Singla AK. 2001. Chemistry and pharmacology of the citrus bioflavonoid hesperidin. Phytother Res 15: 655-669.

2) Galati EM, Trovato A, Kirjavainen S, Forestieri AM, Rossitto A, Monforte MT. 1996. Biological effects of hesperidin, a citrus flavonoid. (Note III): Antihypertensive and diuretic activity in rat. IL Farmaco 51: 219-221.

3) Galati EM, Monforte MT, Kirjavainen S, Forestieri AM, Trovato A, Tripodo MM. 1994. Biological effects of hesperidin, a citrus flavonoid. (Note I): Antiinflammatory and analgesic activity. IL Farmaco 49: 709-712.

4) Hijiya H, Miyake T. 1991. Alpha-glycosyl hesperidin, and its preparation and uses. European Patent Publication No 0402049.

5) Kometani T, Terada Y, Nishimura T, Takii H, Okada S. 1994. Transglycosylation to hesperidin by cyclodextrin glucanotransferase from an alkalophilic Bacillus species in alkaline $\mathrm{pH}$ and properties of hesperidin glycosides. Biosci Biotech Biochem 58: 1990-1994.

6) Yamada M, Mitsuzumi H, Tsuzaki Y, Miwa Y, Chaen H, Yamamoto I. 2003. Antioxidant activity of glycosylated vitamin $\mathrm{P}$ and its suppressive effect on oxidative stress in hyperlipidemic mice. J Jpn Soc Nutr Food Sci 56: 355 363.

7) Ohtsuki K, Abe A, Mitsuzumi H, Kondo M, Uemura K, Iwasaki Y, Kondo Y. 2002. Effects of long-term administration of hesperidin and glucosyl hesperidin to spontaneously hypertensive rats. J Nutr Sci Vitaminol 48: 420422.

8) Ohtsuki K, Abe A, Mitsuzumi H, Kondo M, Uemura K, Iwasaki Y, Kondo Y. 2003. Glucosyl hesperidin improves serum cholesterol composition and inhibits hypertrophy in vasculature. J Nutr Sci Vitaminol 49: 447-450.

9) Nishizaki Y, Tatefuji T, Akamatsu S, Hanaya T, Arai S, Ikeda M, Kurimoto M. 1997. Anti-allergic effect of $\alpha$ glucosyl-hesperidin. Med Biol 135: 199-204.

10) Miwa Y, Yamada M, Sunayama T, Mitsuzumi H, Tsuzaki 
Y, Chaen H, Mishima Y, Kibata M. 2004. Effects of glucosyl hesperidin on serum lipids in hyperlipidemic subjects: preferential reduction in elevated serum triglyceride level. J Nutr Sci Vitaminol 50: 211-218.

11) Manninen V, Tenkanen L, Koskinen P, Huttunen JK, Mänttäri M, Heinonen OP, Frick MH. 1992. Joint effects of serum triglyceride and LDL cholesterol and HDL cholesterol concentrations on coronary heart disease risk in the Helsinki Heart Study. Implications for treatment. Circulation 85: 37-45.

12) Grundy SM, Vega GL. 1992. Two different views of the relationship of hypertriglyceridemia to coronary heart disease. Implications for treatment. Arch Intern Med 152: 28-34.

13) Hodis HN, Mack WJ, Azen SP, Alaupovic P, Pogoda JM, LaBree L, Hemphill LC, Kramsch DM, Blankenhorn DH. 1994. Triglyceride- and cholesterol-rich lipoproteins have a differential effect on mild/moderate and severe lesion progression as assessed by quantitative coronary angiography in a controlled trial of lovastatin. Circulation 90: $42-49$.

14) Feingold KR, Grunfeld C, Pang M, Doerrler W, Krauss RM. 1992. LDL subclass phenotypes and triglyceride metabolism in non-insulin-dependent diabetes. Arterioscler Thromb 12: 1496-1502.

15) Kazumi T, Kawaguchi A, Hozumi T, Nagao M, Iwahashi M, Hayakawa M, Ishihara K, Yoshino G. 1999. Low density lipoprotein particle diameter in young, nonobese, normolipidemic Japanese men. Atherosclerosis 142: $113-119$.

16) McNamara JR, Jenner JL, Li Z, Wilson PWF, Schaefer EJ. 1992. Change in LDL particle size is associated with change in plasma triglyceride concentration. Arterioscler Thromb 12: 1284-1290.

17) Austin MA, Mykkänen L, Kuusisto J, Edwards KL, Nelson C, Haffner SM, Pyörälä K, Laakso M. 1995. Prospective study of small LDLs as a risk factor for noninsulin dependent diabetes mellitus in elderly men and women. Circulation 92: 1770-1778.

18) Nakajima K, Saito T, Tamura A, Suzuki M, Nakano T, Adachi M, Tanaka A, Tada N, Nakamura H, Campos E, Havel RJ. 1993. Cholesterol in remnant-like lipoproteins in human serum using monoclonal anti apo B-100 and anti apo A-I immunoaffinity mixed gels. Clin Chim Acta 223: $53-71$.

19) The Committee on Enzymes of the Scandinavian Society for Clinical Chemistry and Clinical Physiology. 1974. Recommended methods for the determination of four enzymes in blood. Scand J Clin Lab Invest 33: 291-306.

20) Sakai T. 1989. Clinical significance of gamma-glutamyl transpeptidase analysis in biochemical laboratory tests. Nippon Rinsho 48 (Suppl): 277-281.

21) Bowers LD, Wong ET. 1980. Kinetic serum creatinine assays. II. A critical evaluation and review. Clin Chem 26: $555-561$.

22) Sanders GT, Pasman AJ, Hoek FJ. 1980. Determination of uric acid with uricase and peroxidase. Clin Chim Acta 101: 299-303.

23) Mishima $Y$, Ando M, Kuyama Y, Ishioka T, Kibata M. 1997. A simple method for identifying particle size of low density lipoprotein using PAG electrophoresis: comparison between LipoPhor and LipoPrint LDL systems. Jpn Atheroscler Soc 25: 67-70.

24) Kibata M, Kikuti T, Ishii K, Kushida A, Fujita K, Mishima Y. 2002. Fenofibrate treatment changed polyacryl- amide gel electrophoresis (PAGE) patterns of dyslipidemias and their components: a proposal for the clinical usefulness of SEAND classification. Jpn Atheroscler Soc 29: 33-39.

25) Shirai K, Nema T, Hiroh $Y$, Itoh $Y$, Miyashita $Y$, Watanabe H. 1997. Clinical efficacy of the direct assay method using polymers for serum high density lipoprotein cholesterol. J Clin Lab Anal 11: 82-86.

26) Hitsumoto T, Yoshinaga K, Aoyagi K, Sakurai T, Kanai M, Uchi T, Noike H, Ohsawa H, Watanabe H, Shirai K. 2002. Association between preheparin serum lipoprotein lipase mass and acute myocardial infarction in Japanese men. J Atheroscler Thromb 9: 163-169.

27) Ai M, Tanaka A. 2001. Guidelines for plasma lipid and lipoprotein concentration. Nippon Rinsho 59 (Suppl 2): 759-762.

28) Vigna GB, Donega P, Passaro A, Zanca R, Cattin L, Fonda M, Pauciullo P, Marotta G, Fellin R, Gasparrini S, Piliego T. 1999. Post-prandial effects of gemfibrozil vs simvastatin in hypercholesterolemic subjects with borderline hypertriglyceridemia. Nutr Metab Cardiovasc Dis 9: $234-243$.

29) Devaraj S, Vega G, Lange R, Grundy SM, Jialal I. 1998. Remnant-like particle cholesterol levels in patients with dysbetalipoproteinemia or coronary artery disease. $\mathrm{Am}$ J Med 104: 445-450.

30) Takata K. 2001. The metabolic regulation of TG-rich lipoproteins. Nippon Rinsho 59 (Suppl 2): 472-476.

31) Daubresse JC, Machowski R, Pulinx E. 1994. Efficacy of simvastatin for lowering cholesterol in non-insulin dependent diabetic patients with hypercholesterolemia. Acta Clin Belg 49: 68-75.

32) Sone H, Takahashi A, Shimano H, Ishibashi S, Yoshino G, Morisaki N, Saito Y, Kawazu S, Teramoto T, Fujita T, Shiba T, Iwamoto Y, Kuzuya N, Akanuma Y, Yamada N. 2002. HMG-CoA reductase inhibitor decreases small dense low-density lipoprotein and remnant-like particle cholesterol in patients with type-2 diabetes. Life Sci 71: 2403-2412.

33) Andersson Y, Majd Z, Lefebvre AM, Martin G, Sechkin AV, Kosykh V, Fruchart JC, Najib J, Staels B. 1999. Developmental and pharmacological regulation of apolipoprotein C-II gene expression. Comparison with apo C-I and apo C-III gene regulation. Arterioscler Thromb Vasc Biol 19: 115-121.

34) Clavey V, Copin C, Mariotte MC, Baugé E, Chinetti G, Fruchart J, Fruchart JC, Dallongeville J, Staels B. 1999. Cell culture conditions determine apolipoprotein C-III secretion and regulation by fibrates in human hepatoma HepG2 cells. Cell Physiol Biochem 9: 139149 .

35) Weisgraber KH. 1994. Apolipoprotein E: structurefunction relationships. Adv Protein Chem 45: 249-302.

36) Olofsson SO, Asp L, Boren J. 1999. The assembly and secretion of apolipoprotein B-containing lipoproteins. Curr Opin Lipidol 10: 341-346.

37) Boren J, Rustaeus S, Olofsson SO. 1994. Studies on the assembly of apolipoprotein B-100- and B-48-containing very low density lipoproteins in McA-RH7777 cells. J Biol Chem 269: 25879-25888.

38) Wilcox LJ, Barrett PHR, Newton RS, Huff MW. 1999. ApoB 100 secretion from HepG2 cells is decreased by the ACAT inhibitor CI-1011: an effect associated with enhanced intracellular degradation of apoB. Arterioscler Thromb Vasc Biol 19: 939-949. 
39) Haghpassand M, Wilder D, Moberly JB. 1996. Inhibition of apolipoprotein B and triglyceride secretion in human hepatoma cells (HepG2). J Lipid Res 37: 1468-1480.

40) Borradaile NM, Carroll KK, Kurowska EM. 1999. Regulation of HepG2 cell apolipoprotein B metabolism by the citrus flavanones hesperetin and naringenin. Lipids 34: 591-598.

41) Wilcox LJ, Borradaile NM, de Dreu LE, Huff MW. 2001. Secretion of hepatocyte apoB is inhibited by the flavonoids, naringenin and hesperetin, via reduced activity and expression of ACAT2 and MTP. J Lipid Res 42: 725-734.

42) Cha JY, Cho YS, Kim I, Anno T, Rahman SM, Yanagita T. 2001. Effect of hesperetin, a citrus flavonoid, on the liver triacylglycerol content and phosphatidate phosphohydrolase activity in orotic acid-fed rats. Plant Foods Hum Nutr 56: 349-358.

43) Bok SH, Lee SH, Park YB, Bae KH, Son KH, Jeong TS, Choi MS. 1999. Plasma and hepatic cholesterol and hepatic activities of 3-hydroxy-3-methyl-glutaryl-CoA reductase and acyl CoA: cholesterol transferase are lower in rats fed citrus peel extract or a mixture of citrus bioflavonoids. J Nutr 129: 1182-1185.

44) Park YB, Do KM, Bok SH, Lee MK, Jeong TS, Choi MS. 2001. Interactive effect of hesperidin and vitamin E sup- plements on cholesterol metabolism in high cholesterolfed rats. Int J Vitam Nutr Res 71: 36-44.

45) Nozue T, Inazu A, Mabuchi H. 2001. High density lipoprotein. Nippon Rinsho 59 (Suppl 2): 75-79.

46) Okada M. 2001. Functions of cholesteryl ester transfer protein (CETP). Nippon Rinsho 59 (Suppl 2): 236-239.

47) Blackburn P, Cote M, Lamarche B, Couillard C, Pascot A, Tremblay A, Bergeron J, Lemieux I, Despres JP. 2003. Impact of postprandial variation in triglyceridemia on low-density lipoprotein particle size. Metabolism 52: 1379-1386.

48) Hirano T, Oi K, Sakai S, Kashiwazaki K, Adachi M, Yoshino G. 1998. High prevalence of small dense LDL in diabetic nephropathy is not directly associated with kidney damage: a possible role of postprandial lipemia. Atherosclerosis 141: 77-85.

49) Kwiterovich PO Jr. 2002. Clinical relevance of the biochemical, metabolic, and genetic factors that influence low-density lipoprotein heterogeneity. Am J Cardiol 90: $30 \mathrm{i}-47 \mathrm{i}$.

50) Chiba H, Uehara $M$, Wu J, Wang $X$, Masuyama $R$, Suzuki K, Kanazawa K, Ishimi Y. 2003. Hesperidin, a citrus flavonoid, inhibits bone loss and decreases serum and hepatic lipids in ovariectomized mice. J Nutr 133: 1892-1897. 